Results In total 4022 people aged 55 years were invited and 1129 $(28 \%)$ screened over the 3-month period. Screening uptake differed by method of invitation, with a simple approach being significantly more successful than one that was more interactive (32\% vs $27 \%$, $\mathrm{p}=0.0015)$. Uptake also decreased significantly with increasing deprivation. Adenomas were found in 111 (9.8\%) of those screened. Cancer was found in two subjects. Over $95 \%$ of participants rated the procedure as "very" or "fairly" acceptable. Over $90 \%$ of respondents said they would participate in cancer screening in the future and a similar proportion would recommend doing so to others. 11 out of 31 practices offered an estimate of the time spent on activities relating to this pathfinder for FSIG screening, most giving a figure of $<1 \%$.

Conclusion In this pathfinder study, a simple invitation to FSIG achieved a greater uptake rate than a more complex, interactive method of invitation. Deprivation was associated with lower screening uptake. Further pilots of the flexible sigmoidoscopy screening programme should focus on strategies to increase response rate and to improve technical aspects of the procedure. Further evaluation should be incorporated into that development.

Competing interests None declared.

\section{PWE-078 INCIDENCE AND MANAGEMENT OF MALIGNANT POLYPS IN THE NORTH ESSEX BOWEL CANCER SCREENING PROGRAMME}

doi:10.1136/gutjnl-2012-302514d.78

E Paulon,* D O'Riordan, A Shenoy. Department of Gastroenterology, Colchester General Hospital, Colchester, UK

Introduction The outcome of colonic cancer in the UK population has sensibly improved since the introduction of the Bowel Cancer Screening Programme (BCSP) in 2006. This is mainly due to early detection of the cancer in its early stages of which the malignant polyp (MP) is often a manifestation. In this study we reviewed the detection rates and management of malignant polyps and their outcomes in the population of north and mid Essex and compared it to national and international experience.

Methods 33 patients with malignant polyps were identified between the start of the North Essex screening programme (February 2009) and December 2010. Data were obtained using patient notes, endoscopy, radiology and histopathology reports.31 patients were included in the study because data of two patients were not available.

Results Of the 31 polyps, the vast majority (94\%) was located in the left colon. $19(61 \%)$ were peduncolated. Polyps average size was $1.5 \mathrm{~cm}, 8(26 \%)$ measured $>2 \mathrm{~cm}$ and only $2(6 \%)$ measured $<1 \mathrm{~cm}$. Haggitt and Kikuchi classifications were used to assess the depth of invasion. Patients were divided into low risk (LR) and high risk (HR) groups according to histologic criteria (depth of invasion, lymphovascular invasion, grade and excision margins $>2 \mathrm{~mm}$ ). 16 (52\%) fell into the LR group, 15 (48\%) were considered at high risk. 29 (94\%) patients had staging CT, with an average waiting time of 3.5 weeks for patients at HR (range 2-13 weeks) and 6 weeks for patients at LR (range 2-24 weeks). Five patients were surgically treated within 10 weeks (4 HR, $1 \mathrm{LR}$ ). The remaining patients were followed-up with a repeat endoscopy, with a mean waiting time of 9 weeks for patients at HR (range $4-15$ weeks) and 24 weeks for patients at LR (range 9-60 weeks). Residual tumour was identified on endoscopic follow-up in two patients at HR. Surgery was finally performed in 15 patients (48.5\%), $13 \mathrm{HR}, 2 \mathrm{LR}$. Lymph nodes (LNs) involvement was present in four patients (30\%). Residual tumour was found in 5 (33\%). Surgical complications occurred in two patients $(13 \%)$ and consisted in one anastomotic leak and one bilateral pulmonary embolism. To date cancer recurrence has been found in $2 \mathrm{HR}$ patients $(6.5 \%)$ who had evidence of LNs involvement (median follow-up of 16 months, range 8-28).

Conclusion CT scans and endoscopic follow-up timing varied greatly between patients, especially for those in the LR group. LNs involvement and recurrence rate $(30 \%)$ was higher than described in London BCSP centres (9.8\%) but still consistent with other published data. ${ }^{1}$ Further research is needed to clarify the need for staging CT and timing of follow-up endoscopy in patients at LR.

Competing interests None declared.

\section{REFERENCE}

1. Hassan C, Zullo A, Winn S, et al. The colorectal malignant polyp: scoping a dilemma Dig Liver Dis 2007;39:92-100.

\section{PWE-079 CHARACTERISATION COLONIC POLYPS USING FICE WITHOUT OPTICAL MAGNIFICATION: A NEW CLASSIFICATION SYSTEM (N.A.C.)}

doi:10.1136/gutjnl-2012-302514d.79

G Longcroft-Wheaton,* P Bhandari. Department of Gastroenterology, Portsmouth Hospitals NHS Trust, Portsmouth, UK

Introduction FICE is a novel method of electronic imaging on fujinon endoscopes which enhances surface and vascular patterns. It can be used to predict histology of colonic polyps. However, there are no validated classification systems for using the technique without magnification. This study aims to validate a novel tool for this purpose.

Methods The optimum FICE setting was determined in a picture study. Digital photographs were taken of polyps with white light and all 10 FICE settings at $1026 \times 770$ resolution. They were reviewed by two endoscopists who scored each image for clarity of the mucosal and vascular patterns on a 3 point scale $(0=$ poor, $3=$ excellent). A second library was examined to determine the criteria associated with hyperplastic polyps, adenomas and cancer. In each case the true histology was known. A prospective study was then performed to test the identified characteristics in vivo. Finally the criteria were used in a prospective study of polyps $<10 \mathrm{~mm}$ in size within a screening population.

\section{Abstract PWE-079 Table 1}

\begin{tabular}{|c|c|c|c|c|c|c|}
\hline & \multicolumn{2}{|c|}{ Hyperplastic } & \multicolumn{2}{|c|}{ Adenomas } & \multicolumn{2}{|l|}{ Cancers } \\
\hline & Study 2 & Study 3 & Study 2 & Study 3 & Study 2 & Study 3 \\
\hline \multicolumn{7}{|l|}{ WLI features (vascularity) } \\
\hline Pale & $83 \%$ & $59 \%$ & $22 \%$ & $17 \%$ & $0 \%$ & $0 \%$ \\
\hline Normal & $0 \%$ & $4 \%$ & $0 \%$ & $13 \%$ & $0 \%$ & $0 \%$ \\
\hline Dark & $17 \%$ & $37 \%$ & $78 \%$ & $70 \%$ & $100 \%$ & $100 \%$ \\
\hline \multicolumn{7}{|l|}{ FICE features (vascularity) } \\
\hline Pale & $80 \%$ & $67 \%$ & $6 \%$ & $17 \%$ & $0 \%$ & $0 \%$ \\
\hline Dark & $20 \%$ & $33 \%$ & $94 \%$ & $83 \%$ & $0 \%$ & $0 \%$ \\
\hline Very dark & $0 \%$ & $0 \%$ & $0 \%$ & $0 \%$ & $100 \%$ & $100 \%$ \\
\hline \multicolumn{7}{|l|}{ FICE features (vascular patterns) } \\
\hline Absent vascular pattern & $50 \%$ & $48 \%$ & $0 \%$ & $13 \%$ & $0 \%$ & $0 \%$ \\
\hline Faint vessels not following crypts & $50 \%$ & $35 \%$ & $0 \%$ & $1 \%$ & $0 \%$ & $0 \%$ \\
\hline Regular pericryptal pattern & $0 \%$ & $17 \%$ & $94 \%$ & $86 \%$ & $0 \%$ & $0 \%$ \\
\hline Dense, irregular pattern & $0 \%$ & $0 \%$ & $0 \%$ & $0 \%$ & $100 \%$ & $100 \%$ \\
\hline \multicolumn{7}{|l|}{ FICE surface pattern } \\
\hline No surface pattern & $63 \%$ & $37 \%$ & $0 \%$ & $0 \%$ & $0 \%$ & $0 \%$ \\
\hline Large, non-compact crypt pattern & $37 \%$ & $39 \%$ & $0 \%$ & $9 \%$ & $0 \%$ & $0 \%$ \\
\hline Small, compact, regular pattern & $0 \%$ & $17 \%$ & $88 \%$ & $88 \%$ & $0 \%$ & $0 \%$ \\
\hline Disorganised, irregular pattern & $0 \%$ & $0 \%$ & $0 \%$ & $0 \%$ & $100 \%$ & $100 \%$ \\
\hline Cannot assess & $0 \%$ & $7 \%$ & $12 \%$ & $3 \%$ & $0 \%$ & $0 \%$ \\
\hline
\end{tabular}

